\title{
Trendwende am Arbeitsmarkt seit 2005: Jobboom mit Schattenseiten?
}

\author{
Der kräftige Wirtschaftsaufschwung von 2005 bis 2008 setzte eine Trendwende am \\ deutschen Arbeitsmarkt in Gang. Seitdem legte die Erwerbstätigkeit kräftig zu, die \\ Arbeitslosigkeit ging deutlich zurück. Im Zusammenhang mit der verbesserten \\ Beschäftigungssituation werden aber auch Schattenseiten gesehen. Im Vordergrund \\ steht dabei die Ausbreitung flexibler und schlecht entlohnter Beschäftigungsformen. \\ Der Beitrag will auf der Basis empirischer Fakten einen genauen Blick auf die struktu- \\ relle Zusammensetzung der Erwerbstätigkeit in der jüngeren Vergangenheit werfen. \\ Um die Tragweite der Trends besser einordnen zu können, werden zudem Befunde \\ zu Aufstiegschancen aus weniger stabilen oder nicht voll existenzsichernden \\ Beschäftigungsverhältnissen diskutiert.
}

CARINA SPERBER, ULRICH WALWEI

\section{Einleitung}

Deutschland galt nach der Wiedervereinigung bis etwa Mitte der letzten Dekade als „kranker“ Mann Europas. Das Wirtschaftswachstum war seinerzeit vergleichsweise gering, die Erwerbstätigkeit entwickelte sich nur schwach und die Arbeitslosigkeit stieg von Rezession zu Rezession. Spätestens seit den eher glimpflichen Folgen der schweren Wirtschafts- und Finanzkrise 2008/2009 änderte sich die Wahrnehmung in der Öffentlichkeit. Seitdem macht das sogenannte „Deutsche Beschäftigungswunder“ die Runde. Denn unerwarteterweise konnte selbst ein in der Bundesrepublik noch nie da gewesener Rückgang des Bruttoinlandsprodukts von - 5,6\% im Jahr 2009 dem Arbeitsmarkt nur wenig anhaben. Die dahinter liegende Trendwende am Arbeitsmarkt setzte hierzulande aber vorher ein, etwa Mitte des letzten Jahrzehnts. Mit dem kräftigen Wirtschaftsaufschwung von 2005 bis 2008 und dem damit verbundenen außerordentlich starken Beschäftigungswachstum ging die Arbeitslosigkeit erstmals seit langer Zeit spürbar zurück. Der positive Beschäftigungstrend hält auch heute noch an.

Oft wird demgegenüber eingewandt, dass mit der verbesserten „Großwetterlage“ am Arbeitsmarkt Schattenseiten verbunden und daraus neue Probleme entstanden seien. Die Trendwende sei mit einer veränderten und insgesamt ungünstigeren Struktur der Beschäftigung einher- gegangen. Dies habe zu mehr Ungleichheit in der Beschäftigung beigetragen. Dahinter steht die Beobachtung eines Zuwachses an atypischen und/oder niedrig entlohnten Beschäftigungsformen, die tendenziell mit weniger Stabilität und einem geringeren sozialen Schutz verbunden sein können. So stellt der Deutsche Gewerkschaftsbund (DGB) fest, dass den aktuellen Wandel der Arbeitswelt ein schleichender Bedeutungsverlust des Normalarbeitsverhältnisses begleite (DGB 2013). In den Veränderungen zeige sich eine Auflösung der Arbeitsmarktordnung der Nachkriegszeit (Bosch 2012). Auch auf internationaler Ebene wird ähnliche Kritik laut. Der Präsident des in Washington ansässigen Peterson Institute for International Economics schreibt, dass die hohe Wettbewerbsfähigkeit Deutschlands durch niedrige Löhne und eine Zunahme prekärer Arbeitsverhältnisse erkauft worden sei (Posen 2013). Selbst die OECD sieht den stark gewachsenen Niedriglohnsektor und den hohen Anteil befristet Beschäftigter in Deutschland als problematisch (OECD 2014).

Die Frage ist, ob und inwieweit sich die kritische Sicht auf die Entwicklungstendenzen in der Zusammensetzung der Beschäftigung bei einer genaueren Betrachtung als berechtigt erweisen. Deswegen will der Beitrag zwei Aspekten in besonderer Weise nachgehen: Zum einen geht es uns um die strukturelle Zusammensetzung der Erwerbstätigkeit seit 2005, insbesondere mit Blick auf spezifische Aspekte der Beschäftigung wie Arbeitszeit, Beschäf- 
ABB. 1

\section{Entwicklung der Arbeitslosigkeit von 1992 bis zum aktuellen Rand* \\ Angaben in Prozent}

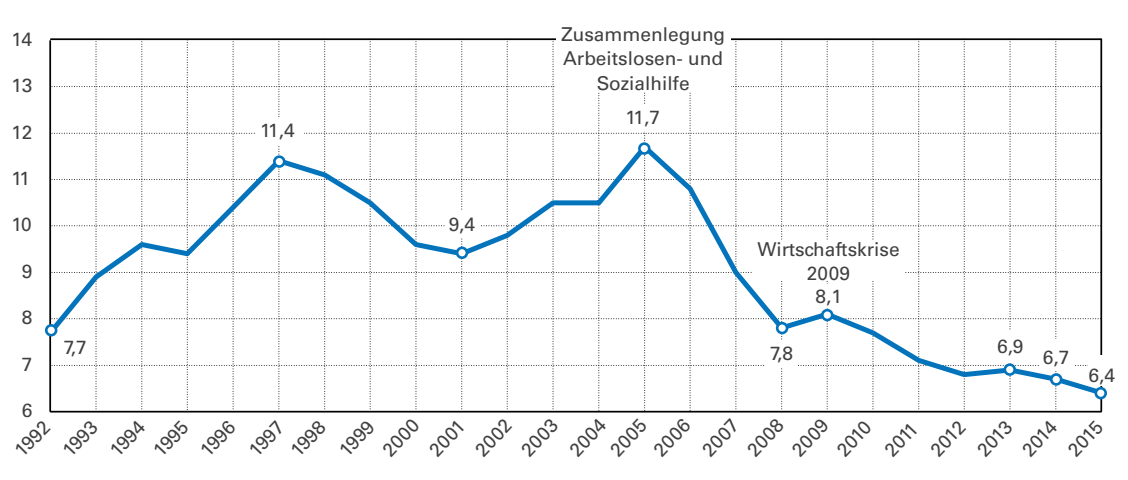

*Arbeitslosenquote (bezogen auf alle zivilen Erwerbspersonen) in \%, Deutschland, 1992 bis 2013, IAB Prognose für 2014/2015. Zitierhinweis: Statistik der Bundesagentur für Arbeit, Arbeitslosigkeit im Zeitverlauf.

Quelle: Bundesagentur für Arbeit, Arbeitsmarktberichterstattung Daten: Statistik der BA, Prognose: IAB. tigungssicherheit, Entlohnung und Existenzsicherung. Zum anderen wird das Tempo von Veränderungen in den Jahren mit verbesserter Arbeitsmarktlage ab 2005 betrachtet und danach gefragt, ob sich dieses - verglichen mit der noch durch eine schlechtere Arbeitsmarkt-Performance gekennzeichneten Vorperiode - in der jüngeren Vergangenheit deutlich beschleunigt hat. Um weitere Anhaltspunkte für eine Einschätzung der jüngeren Entwicklung zu bekommen, werden zusätzlich Befunde zu Aufstiegschancen aus weniger stabilen und nicht oder nur begrenzt existenzsichernden Beschäftigungsverhältnissen herangezogen.

\section{Aufschwung am Arbeitsmarkt}

Die längerfristige Entwicklung am Arbeitsmarkt offenbart, dass sich Mitte der letzten Dekade ein Umschwung am Arbeitsmarkt vollzogen hat (Abbildung 1). Seit den 1970er Jahren stieg die Arbeitslosenquote zunächst von Rezession zu Rezession. Offenbar war kein wirtschaftlicher Aufschwung in der Lage, die Arbeitslosigkeit wieder auf das Niveau vor der letzten Rezession zurückzuführen. Der strukturelle Sockel der Arbeitslosigkeit baute sich so Zug um Zug auf. In der Literatur spricht man in diesem Zusammenhang von sogenannten „Hysterese-Effekten“ (Möller/Völker 1991; Belke/Göcke 1994). Seit 2005 änderte sich das Bild grundlegend. Begünstigt durch die verbesserte Aufnahmefähigkeit des Arbeitsmarktes in Folge der „Hartz-Reformen“ und eine nicht zuletzt durch die hohe Wettbewerbsfähigkeit des Verarbeitenden Gewerbes insgesamt verbesserte wirtschaftliche Entwick- lung verringerte sich die Arbeitslosigkeit seit 2005 um rund $40 \%$ (2 Mio.) oder gut fünf Prozentpunkte (Walwei 2011). Auch die moderate Lohnpolitik in Zeiten des Booms dürfte den Beschäftigungsaufschwung begünstigt haben. Dustmann et al. (2014) nennen verschiedene Gründe für die Lohnzurückhaltung auf gesamtwirtschaftlicher Ebene. Dazu zählen sie die schwierige Lage von Wirtschaft und Arbeitsmarkt in den 1990er Jahren und zu Beginn des neuen Jahrtausends, die eine Reformbereitschaft erzeugt hätten. Angesprochen werden zudem die abnehmende Tarifbindung und der gesunkene gewerkschaftliche Organisationsgrad. Schließlich verweisen die Autoren als Ursache für die schwache Reallohnentwicklung auf die Spätfolgen der finanziellen Lasten nach der Wiedervereinigung.

Die Erwerbstätigkeit erreichte im Jahr 2013 mit jahresdurchschnittlich 42,3 Mio. ein Allzeithoch. Die gesamtwirtschaftliche Zahl der geleisteten Arbeitsstunden (Arbeitsvolumen der Erwerbstätigen) lag zwar zuletzt noch nicht wieder auf dem Wert zu Beginn der 1990er Jahre, jedoch stieg sie zwischen 2005 und 2013 und damit im Zuge des Aufschwungs um knapp 4\% von 55,50 Mrd. auf 57,61 Mrd. Arbeitsstunden spürbar an (Fuchs et al. 2014). Am aktuellen Rand setzt sich der positive Beschäftigungstrend kontinuierlich fort. Somit ist in den letzten Jahren eine substanzielle Verbesserung am Arbeitsmarkt nicht zu übersehen.

Dennoch ist die Situation am Arbeitsmarkt insgesamt noch nicht zufriedenstellend. Für bestimmte Gruppen, wie z. B. Langzeitarbeitslose, ist es angesichts der zuletzt wieder stagnierenden Arbeitslosigkeit schwer, einen $\mathrm{Zu}$ gang zum Arbeitsmarkt zu finden. Die Arbeitslosenquote ist auf der Bundesebene noch weit von dem als üblich unterstellten Vollbeschäftigungsniveau von 3-4\% entfernt (Dietz et al. 2012; Weber 2014). Insbesondere regional zeigen sich weiterhin enorme Disparitäten mit Blick auf die jeweilige Arbeitsmarktsituation. Beispielsweise unterscheiden sich die Arbeitslosenquoten immer noch gravierend zwischen Ost- (10,3\%) und West-Deutschland $(6,0 \%)$. So verzeichneten Bayern (3,8\%) und BadenWürttemberg (4,1\%) im Jahr 2013 die niedrigsten Arbeitslosenquoten, wohingegen Berlin (11,7\%) und Mecklenburg-Vorpommern (11,7\%) die höchsten Arbeitslosenquoten aufwiesen (Statistik der Bundesagentur für Arbeit 2014a).

Des Weiteren sind Entwicklungen in der Struktur der Beschäftigung zu konstatieren, die nicht selten mit mehr Ungleichheit in der Beschäftigung in Verbindung gebracht werden (Brehmer/Seifert 2008; Keller/Seifert 2013). Dies verweist auf einen altbekannten Zielkonflikt zwischen der Quantität und der Qualität von Beschäftigung. Danach sind hohe Arbeitslosigkeit und ein beträchtlicher Anteil instabiler und nicht existenzsichernder Beschäftigung gleichzeitig nur schwer zu vermeiden, weil sie als zwei Seiten derselben Medaille gesehen werden können (Krugman 1994). 


\section{Veränderungen in der Beschäftigungs- struktur und potenzielle Risiken}

Bei den vieldiskutierten Veränderungen in der Zusammensetzung der Beschäftigung stehen zwei Merkmale im Fokus. Erstens geht es um den Bedeutungsverlust des sog. „Normalarbeitsverhältnisses“, also den Rückgang unbefristeter Vollzeitbeschäftigung außerhalb der Zeitarbeitsbranche, sowie den aufwärtsgerichteten Trend davon abweichender sog. atypischer Erwerbsformen, wie z. B. befristete Beschäftigung, geringfügige Beschäftigung, Leiharbeit und Teilzeitbeschäftigung in den letzten Jahren (Dietz et al. 2013). Eine zweite Dimension betrifft darüber hinausgehende Strukturmerkmale der Beschäftigung, die wie die Entlohnung quer zu den Erwerbsformen liegen können. Berücksichtigt man solche Strukturmerkmale und Arbeitnehmerpräferenzen, sind sowohl Normalarbeitsverhältnisse als auch atypische Beschäftigungsverhältnisse differenziert zu betrachten. Daraus folgt, dass atypische Beschäftigung nicht mit „prekären“ Arbeitsverhältnissen gleichgesetzt werden darf. Denn „Prekarität“ zielt im Kern auf eine dauerhaft eingeschränkte Existenzsicherung durch Arbeit während des Erwerbslebens und im Alter ab. Prekär Beschäftigte besitzen zudem nur geringe Arbeitsplatzsicherheit, haben wenig Einfluss auf die Ausgestaltung ihrer Arbeitssituation und können nur sehr eingeschränkt arbeitsrechtlichen Schutz in Anspruch nehmen (Klenner 2011; Rodgers 1989). Um dem Rechnung zu tragen, nehmen wir in unserer Untersuchung die Zahl und die relative Bedeutung der Beschäftigten mit niedrigen Stundenverdiensten mit ins Blickfeld (Kalina/Weinkopf 2014). Zudem gilt unser Augenmerk den Personen, die aufgrund zu geringer Erwerbseinkünfte in ihrem jeweiligen Haushaltskontext kein existenzsicherndes Einkommen erreichen und deshalb als sog. "Aufstocker" ergänzende Grundsicherungsleistungen beziehen (Bruckmeier et al. 2013).

Die beobachteten Verschiebungen in den Beschäftigungsstrukturen bergen zwar potenzielle Risiken wachsender Ungleichheit, dennoch handelt es sich hier nicht um einen Automatismus. Hierfür gibt es drei Gründe: Erstens weisen atypische Erwerbsformen ein hohes Maß an Heterogenität auf. So kann insbesondere eine freiwillig gewählte Teilzeitbeschäftigung ${ }^{1}$ die Vereinbarkeit von Erwerbsarbeit mit anderen Aktivitäten wie Familienarbeit oder Bildung erleichtern. Zweitens stellt sich aus individueller Perspektive immer die Frage, was als Referenz für das ausgeübte Beschäftigungsverhältnis herangezogen wird. So dürften atypische Beschäftigung oder auch eine Niedriglohntätigkeit subjektiv immer noch der Arbeitslosigkeit vorzuziehen sein, weil Letztere das geringste Niveau an gesellschaftlicher Teilhabe aufweist und damit mit einem besonders hohen Risiko der Ungleichheit einhergeht (Hohendanner/Gundert 2011). Drittens darf gerade bei der Frage der Beurteilung einer Beschäftigung die dynamische Perspektive nicht außer Acht gelassen werden. Niedrige
Barrieren für eine Beschäftigung können den Einstieg in den Arbeitsmarkt erleichtern. Atypische Beschäftigung kann eine Alternative zu Arbeitslosigkeit darstellen und als Sprungbrett für Übergänge in reguläre Beschäftigung fungieren. Andererseits können unsichere Arbeitsverträge oder gering entlohnte Beschäftigungsverhältnisse - insbesondere über einen längeren Zeitraum - prekäre Erwerbsverläufe fördern. Von entscheidender Bedeutung für die Beurteilung verschiedener Beschäftigungsformen ist also in erster Linie die Frage, ob Personen, die sich in dem Segment instabiler und nur bedingt existenzsichernder Biografien bewegen, dort längerfristig verharren (müssen), von Drehtüreffekten zwischen Arbeitslosigkeit und Beschäftigung betroffen sind oder hierdurch den Sprung in eine nachhaltige und existenzsichernde Beschäftigung schaffen.

Im Folgenden soll nun die Zusammensetzung der Beschäftigung genauer betrachtet werden. Die im Nachgang verwendeten Zahlen für die detaillierte Beschreibung einzelner Erwerbsformen finden sich in Tabelle 1. Sie stützen sich auf drei Quellen: die Volkswirtschaftliche Gesamtrechnung, die Statistik der Bundesagentur für Arbeit und den Mikrozensus des Statistischen Bundesamtes. ${ }^{2}$

\subsection{Selbstständigkeit: Dynamik hält sich in Grenzen}

Im Zuge des Aufschwungs am Arbeitsmarkt hat die Selbstständigkeit leicht zugelegt. Die Zahl der Selbstständigen (ohne mithelfende Familienangehörige) stieg laut Volkswirtschaftlicher Gesamtrechnung zwischen 2005 und 2013 um 47.000 oder 1,1\%. Der Zuwachs fiel damit deutlich geringer aus als bei der abhängigen Beschäftigung, die im selben Zeitraum ein Wachstum von $8,3 \%$ verzeichnete. Verglichen mit den Jahren vor dem Arbeitsmarktaufschwung (1997/2005: + 12,8 \%) nahm die Wachstumsdynamik der Selbstständigkeit merklich ab. Dabei weist der Mikrozensus zwischen 2005 und 2013 bei den selbstständigen Arbeitgebern (mit Beschäftigten) ein Plus von 3,7\% aus, während sich bei den sog. „Solo-Selbstständigen“ ein leichtes Minus (-0,7 \%) ergab. Der Anstieg der Solo-Selbstständigen vollzog sich vor allem in der Zeit vor 2005 und lag damit zeitlich vor der Trendwende am Arbeitsmarkt. Eine Rolle spielten dabei auch die Hartz-Reformen (2003 bis 2005). Arbeitsmarktpolitische Förderprogramme wie die sogenannte Ich-AG haben der Selbstständigkeit Schub gegeben (Caliendo et al. 2012). Somit lässt sich feststel-

1 Zur relativen Bedeutung der „freiwilligen“ Teilzeitbeschäftigung vgl. Abschnitt 3.4.

2 Durch verschiedene Erfassungs- und Erhebungsmethoden ergeben sich teilweise Niveauunterschiede in den Zeitreihen derTabelle 1, die aber wegen der hier im Vordergrund stehenden Betrachtung über die Zeit keine große Bedeutung haben. 
TABELLE 1

Entwicklung der Erwerbstätigkeit und verschiedener Erwerbs- und Beschäftigungsformen, 1997,2005 und 2013

Angaben in absoluten Zahlen und in Prozent

\begin{tabular}{|c|c|c|c|c|c|c|c|c|c|}
\hline & 1997 & 2005 & 2013 & $\begin{array}{l}1997 / \\
2005\end{array}$ & $\begin{array}{c}2005 / \\
2013\end{array}$ & $\begin{array}{l}\text { Anteil an } \\
\text { allen... }\end{array}$ & 1997 & 2005 & 2013 \\
\hline & \multicolumn{3}{|c|}{ Absolute Zahlen in Tsd. } & \multicolumn{3}{|c|}{ Wachstumsraten in \% } & \multicolumn{3}{|c|}{ Anteil in \% } \\
\hline \multicolumn{10}{|l|}{ Volkswirtschaftliche Gesamtrechnung } \\
\hline Erwerbstätige & 37.947 & 39.326 & 42.281 & 3,6 & 7,5 & ET & 100,0 & 100,0 & 100,0 \\
\hline Selbstständige & 3.911 & 4.410 & 4.457 & 12,8 & 1,1 & ET & 10,3 & 11,2 & 10,5 \\
\hline Abhängig Beschäftigte & 34.036 & 34.916 & 37.824 & 2,6 & 8,3 & ET & 89,7 & 88,8 & 89,5 \\
\hline Arbeitsvolumen in Mio. Stunden & 47.947 & 46.215 & 48.833 & $-3,6$ & 5,7 & & & & \\
\hline \multicolumn{10}{|l|}{ Bundesagentur für Arbeit } \\
\hline Sozialversicherungspflichtige Beschäftigte* & 27.280 & 26.230 & 29.616 & $-3,8$ & 12,9 & $A B$ & 80,2 & 75,1 & 78,3 \\
\hline Geringfügig Beschäftigte insgesamt* & k. A. & 6.335 & 7.399 & k. A. & 16,8 & $A B$ & k. A. & 18,1 & 19,6 \\
\hline Ausschließlich geringfügig Beschäftigte* & k. A. & 4.846 & 5.066 & k. A. & 4,5 & $A B$ & k. A. & 13,9 & 13,4 \\
\hline Geringfügig Beschäftigte im Nebenjob* & k. A. & 1.489 & 2.333 & k. A. & 56,7 & $A B$ & k. A. & 4,3 & 6,2 \\
\hline Aufstocker & k. A. & k. A. & 1.310 & k. A. & k. A. & ET & k. A. & k. A. & 3,1 \\
\hline Leiharbeitnehmer* & 213 & 453 & 852 & 112,7 & 88,1 & SVP & k. A. & 1,7 & 2,9 \\
\hline \multicolumn{10}{|l|}{ Mikrozensus } \\
\hline Erwerbstätige & 35.804 & 36.566 & 39.617 & 2,1 & 8,3 & ET & 100,0 & 100,0 & 100,0 \\
\hline Selbstständige insgesamt & 3.528 & 4.080 & 4.131 & 15,6 & 1,3 & ET & 9,9 & 11,2 & 10,4 \\
\hline Arbeitgeber & 1.776 & 1.789 & 1.855 & 0,7 & 3,7 & ET & 5,0 & 4,9 & 4,7 \\
\hline Ein-Personen-Selbstständige & 1.752 & 2.291 & 2.276 & 30,8 & $-0,7$ & ET & 4,9 & 6,3 & 5,7 \\
\hline Abhängig Beschäftigte (ohne mith. FA) & 29.760 & 30.100 & 33.550 & 1,1 & 11,5 & ET & 83,1 & 82,3 & 84,7 \\
\hline Normalarbeitsverhältnisse & 22.018 & 19.683 & 21.145 & $-10,6$ & 7,4 & $A B$ & 74,0 & 65,4 & 63,0 \\
\hline $\begin{array}{l}\text { Teilzeitbeschäftigte < } 32 \text { Std./Woche (ohne } \\
\text { geringfügig Beschäftigte) }\end{array}$ & 4.401 & 5.209 & 6.504 & 18,4 & 24,9 & $A B$ & 14,8 & 17,3 & 19,4 \\
\hline Ausschließlich geringfügig Beschäftigte & 1.642 & 3.113 & 3.736 & 89,6 & 20,0 & $A B$ & 5,5 & 10,3 & 11,1 \\
\hline Befristet Beschäftigte insgesamt & 1.955 & 2.498 & 2.524 & 27,8 & 1,0 & $A B$ & 6,6 & 8,3 & 7,5 \\
\hline Befristet Beschäftigte $>31$ Std./Woche & 1.541 & 1.798 & 1.735 & 16,7 & $-3,5$ & $A B$ & 5,2 & 6,0 & 5,2 \\
\hline
\end{tabular}

Sozio-oekonomisches Panel

Beschäftigte mit einem niedrigen

Stundenlohn

* Stichtag 30. Juni.

${ }^{1}$ Stand 2012.

Legende: $A B=$ Abhängig Beschäftigte, $E T=$ Erwerbstätige, SVP= Sozialversicherungspflichtig Beschäftigte.

Quelle: Arbeitnehmerüberlassungsstatistik der Bundesagentur für Arbeit ANÜ (2014); Data Warehouse der Bundesagentur für Arbeit (2014); Mikrozensus (2014);

Sozio-oekonomisches Panel SOEP (2012); Volkswirtschaftliche Gesamtrechnung VGR (2014).

len, dass die Selbstständigkeit zwar insgesamt am Arbeitsmarktaufschwung partizipieren konnte. Jedoch hat sich der Strukturwandel zugunsten dieser Erwerbsform seit 2005 erheblich verlangsamt. Dabei muss offen bleiben, ob die in der jüngeren Vergangenheit geringer ausfallende Wachstumsrate der Selbstständigen eher verbesserte Zugangschancen in abhängige Beschäftigung oder aber verminderte Erwerbsaussichten in der Selbstständigkeit reflektiert.

Von besonderer Relevanz für die Frage nach Umschichtungen in der Beschäftigung sind die Solo-Selbstständigen, weil sie im Vergleich zu abhängig Beschäftigten oder Arbeitgebern tendenziell höheren Risiken ausgesetzt sind. Im Gegensatz zu abhängig Beschäftigten müssen sie Beiträge zur Kranken- und Rentenversicherung eigenverantwortlich fi- nanzieren. Und anders als Selbstständige mit Beschäftigten können sie Arbeitsausfälle nicht so leicht kompensieren (Statistisches Bundesamt 2012). Mit Blick auf den Zusammenhang von niedrigem Erwerbseinkommen und dem Erhalt von Transferleistungen kommen Koller et al. (2012) zu dem Ergebnis, dass knapp 3 \% aller Selbstständigen Leistungen aus der Grundsicherung beziehen. Die meisten selbstständigen Aufstocker sind dabei Solo-Selbstständige. Ähnlich wie bei abhängig beschäftigten Aufstockern verlässt auch bei den selbstständigen Aufstockern gut ein Viertel nach einem Jahr den Leistungsbezug (siehe auch Abschnitt 3.9). Dennoch gibt es auch einen „harten Kern“ unter den selbstständigen Aufstockern, die über mehrere Jahre im Leistungsbezug bleiben. 


\subsection{Sozialversicherungspflichtige Beschäftigung: kräftiger Beitrag zum Aufschwung}

Der starke Zuwachs der abhängigen Beschäftigung zwischen 2005 und 2013 von 2,91 Mio. geht rein rechnerisch ausschließlich auf den noch kräftigeren Anstieg der sozialversicherungspflichtigen Beschäftigung (3,39 Mio.) zurück. In der jüngeren Entwicklung der sozialversicherungspflichtigen Beschäftigung zeigt sich zudem eine bemerkenswerte Richtungsänderung. Denn bis zur Mitte der letzten Dekade war ein Rückgang der sozialversicherungspflichtigen Beschäftigung zu verzeichnen (Abbildung 2). Offenkundig ist die sozialversicherungspflichtige Beschäftigung damit der wesentliche Treiber der Trendwende am Arbeitsmarkt. Hierdurch erwerben mehr Menschen Ansprüche auf soziale Absicherung. Dabei darf nicht übersehen werden, dass sozialversicherungspflichtige Beschäftigung ausgesprochen heterogen ist. Darin enthalten sind Teilzeitbeschäftigungsverhältnisse, befristete Arbeitsverträge, Leiharbeitnehmer und Vollzeitbeschäftigte mit niedrigen Stundenlöhnen. Insbesondere die reguläre Teilzeitbeschäftigung leistete einen überproportionalen Beitrag zum Aufwuchs der sozialversicherungspflichtigen Beschäftigung (Statistik der Bundesagentur für Arbeit 2014b).

\subsection{Normalarbeitsverhältnis: Bedeutungsverlust lässt nach}

Im Wesentlichen wurde der Umschwung am Arbeitsmarkt durch die abhängige, insbesondere sozialversicherungspflichtige Beschäftigung getrieben. Darunter sind mit über $60 \%$ die sog. „Normalarbeitsverhältnisse“ noch immer die dominante Beschäftigungsform. Ähnlich wie der Sachverständigenrat zur Begutachtung der gesamtwirtschaftlichen Entwicklung sowie Dietz et al. definieren wir hier das Normalarbeitsverhältnis als ein unbefristetes, unselbstständiges und vollzeitnahes Beschäftigungsverhältnis (mit einer Arbeitszeit von mehr als 31 Stunden in der Woche) außerhalb der Zeitarbeitsbranche (Dietz et al. 2013). Dahinter steht die Überlegung, dass vollzeitnahe Tätigkeiten die Erwerbsarbeit in den Lebensmittelpunkt stellen. Legt man diese Definition zugrunde, wuchs das Normalarbeitsverhältnis zwischen 2005 und 2013 um 1,46 Mio. oder 7,4\%. Zwar setzte sich im Arbeitsmarktaufschwung der Trend des relativen Bedeutungsverlustes des Normalarbeitsverhältnisses mit minus 2,4 Prozentpunkten fort, allerdings mit einem erkennbar schwächeren Tempo als vor der Trendwende am Arbeitsmarkt (minus 8,6 Prozentpunkte). Ein - verglichen mit der Vergangenheit - wachsender Bedeutungsverlust unbefristeter und vollzeitnaher Beschäftigungsverhältnisse ist seit 2005 somit nicht zu erkennen. Eine Ursachenanalyse ist an dieser Stelle schwierig. Ein wesentlicher Grund hierfür könnte aber sein, dass der Push-Faktor Arbeitslosigkeit weniger wirkt und damit Beschäftigte durch die verbesserte Arbeitsmarktsituation wieder über mehr Alternativen verfügen. Jedoch ist dabei zu bedenken, dass auch eine unbefristete, vollzeitnahe Tätigkeit nicht immer armutsvermeidend ist, wenn die Entlohnung
ABB. 2

Entwicklung der sozialversicherungspflichtigen Beschäftigung, 1992 - 2013 Angaben in Tausend

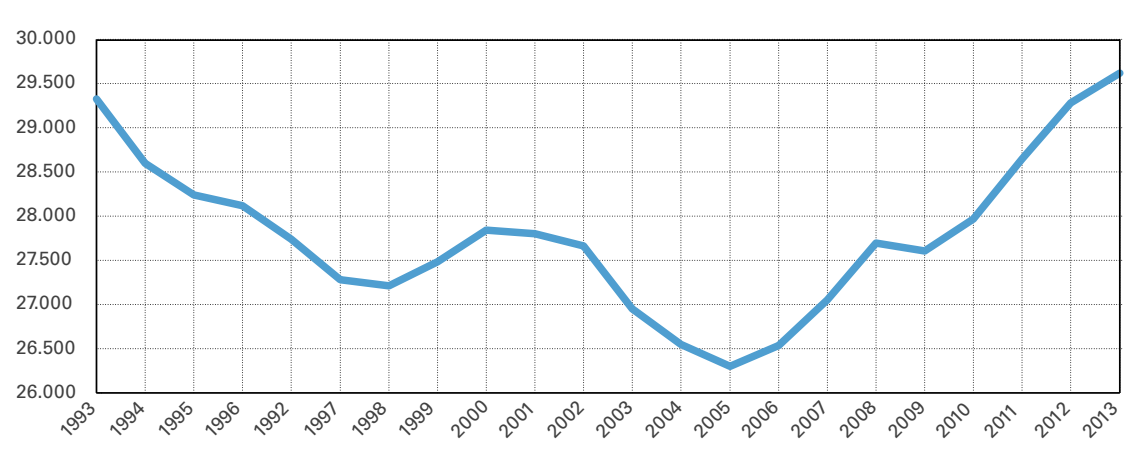

Quelle: Beschäftigtenstatistik der Bundesagentur für Arbeit.

im Niedriglohnbereich angesiedelt ist und andere Einkommen im Haushaltskontext nicht zur Verfügung stehen (Andreß/Seek 2007).

\subsection{Sozialversicherungspflichtige Teilzeit: kontinuierliches Wachstum}

Ähnlich wie bei der Definition von Normalarbeitsverhältnissen stellt sich auch hier zunächst die Frage nach einer geeigneten Abgrenzung zwischen sozialversicherungspflichtiger Voll- und Teilzeitbeschäftigung. So könnte man zunächst einmal jede Abweichung der Wochenarbeitszeit nach unten von der tarifüblichen Vollzeit der Teilzeitbeschäftigung zurechnen, was dann auch für vollzeitnahe Formen gelten würde. ${ }^{3}$ Um dem Rechnung zu tragen, bezieht sich unsere folgende Darstellung auf die bereits oben erwähnte 31-Stunden-Grenze (Dietz et al 2013).

Legt man diese Definition zugrunde, wuchs die - um vollzeitnahe und geringfügige Formen der Beschäftigung bereinigte - Teilzeitbeschäftigung gemessen an der abhängigen Beschäftigung überproportional. Zwischen 2005 bis 2013 war das Wachstum mit 24,9\% nochmals größer als in den Jahren vor 2005 (18,4\%). Eine wichtige Frage für die Beurteilung des Anstiegs ist, inwieweit Teilzeitbeschäftigte am aktuellen Rand vermehrt nach Alternativen suchen als vormals. Der Mikrozensus liefert keine Hinweise auf einen zuletzt stark wachsenden Anteil von Teilzeitbeschäftigten, die eine andere oder zusätzliche Tätigkeit suchen. Das Motiv „Teilzeitbeschäftigung, da Vollzeittätigkeit nicht zu

3 Im Gegensatz dazu wählt das Statistische Bundesamt in seiner Definition einen eher niedrigen Schwellenwert von 20 Arbeitsstunden in der Woche (Wingerter 2009). 
finden“ ist für die hier gewählte Abgrenzung zwischen 2000 (14\%) und 2005 (21,7\%) zwar gestiegen. Mit der Trendwende am Arbeitsmarkt ging der Anteil aber trotz wachsender Teilzeitbeschäftigung sowohl absolut als auch relativ zurück und lag im Jahr 2013 mit 15,3\% fast beim Ausgangsniveau von $2000 .{ }^{4}$ Hieraus sollte aber nicht der voreilige Schluss gezogen werden, dass der realisierte Umfang der Teilzeitbeschäftigung für die Betroffenen immer die erste Wahl darstellen muss. So ist aus Befragungen bekannt, dass insbesondere Frauen mit einer eher geringen wöchentlichen Arbeitszeit gerne mehr arbeiten würden, aber dabei nicht immer eine Vollzeitbeschäftigung anstreben (Wanger 2011; Holst/Seifert 2012). Offen muss zudem bleiben, ob teilzeitbeschäftigte Frauen auch dann nur zu einem geringen Anteil eine zumindest vollzeitnahe Beschäftigung anstreben würden, wenn - aufgrund verbesserter Betreuungsangebote für Kinder und Pflegebedürftige - familiäre Gründe seltener einer spürbaren Ausdehnung der Arbeitszeit entgegenstünden. So deuten neuere Studien darauf hin, dass die vergleichsweise geringen Übergänge von Teilzeitbeschäftigten in ein Normalarbeitsverhältnis nur zum Teil auf arbeitnehmerseitige Arbeitszeitpräferenzen und geschlechtsspezifische Effekte der Haushaltszusammensetzung zurückzuführen sind (Brülle 2013). Insgesamt liefert die jüngere Entwicklung der sozialversicherungspflichtigen Teilzeitbeschäftigung keine wesentlichen Indizien dafür, dass diese einer zunehmenden und nicht intendierten Ungleichheit in der Beschäftigung Vorschub geleistet hätte. Allerdings sind angesichts der kontinuierlich wachsenden Teilzeitbeschäftigung längerfristige Aspekte stärker zu bedenken. So werden bei anhaltender Teilzeitbeschäftigung geringere Rentenanwartschaften erzielt. Von daher sind betriebliche, tarifliche und arbeitsmarktpolitische Möglichkeiten einer Förderung gewünschter Übergänge von Teilzeitbeschäftigten in vollzeitnahe Beschäftigungsformen noch mehr auszuloten.

\subsection{Geringfügige Beschäftigung: meistens Zusatzverdienst, selten Brücke}

Je nach Personengruppe birgt geringfügige Beschäftigung mehr oder weniger große individuelle Risiken. Sie resultieren daraus, dass soziale Absicherung nicht obligatorisch ist und die Entlohnung durch eine monatliche Verdienstgrenze gedeckelt wird. Bei geringfügiger Beschäftigung (Minijobs) ist grundsätzlich zwischen zwei Formen zu unterscheiden: der ausschließlich geringfügigen Beschäftigung und der geringfügigen Beschäftigung im Nebenjob.

Bei ausschließlichen Minijobs zeigt sich nach den 2003 durchgeführten Arbeitsmarktreformen und der damit verbundenen erleichterten Nutzung ein zunächst kräftiger Anstieg. Nach der Trendwende am Arbeitsmarkt ab 2005 ist nach den Daten der Bundesagentur für Arbeit mit einem Plus von 220.000 (+4,5\%) weiterhin ein Anstieg zu verzeichnen. Aufgrund des noch stärkeren Anstiegs der abhängigen Beschäftigung insgesamt $(+8,3 \%)$ nahm die relative Bedeutung der ausschließlich geringfügigen Beschäftigung zwischen
2005 und 2013 von 13,9 auf 13,4\% jedoch ab. Aus diesem Grund ergibt sich auch ein unterproportionaler Beitrag der ausschließlich in Minijobs tätigen Beschäftigten zum Beschäftigungsaufwuchs seit 2005.

Was mögliche Risiken der ausschließlich geringfügigen Beschäftigung auf individueller Ebene angeht, geben die in Minijobs tätigen Personengruppen einen gewissen Aufschluss. Eine Registerumfrage aus dem Jahr 2010 zeigt, dass sich die ausschließlich geringfügige Beschäftigung auf vier Gruppen konzentriert (Körner et al. 2013): Hausfrauen und -männer (35\%), Schülerinnen und Schüler, Studierende (20\%), Rentnerinnen und Rentner (22\%) und Arbeitslose (11\%). Für Schüler, Studierende und Rentner dürfte bei den Minijobs der Zusatzverdienst eindeutig im Vordergrund stehen und ein Wechsel in eine sozialversicherungspflichtige Beschäftigung in der Regel kein Thema sein. Anders stellt sich die Situation aber für Hausfrauen (und die wenigen Hausmänner) und Arbeitslose dar. Überproportional sind Frauen in dieser Beschäftigungsform zu finden. Neben einem Anteil an Frauen (mit und ohne Kinder), die einen Minijob als $\mathrm{Zu}$ verdienst zu einem erwerbstätigen Partner ausüben und deren Arbeitszeitwünsche mit dem aktuellen Minijob übereinstimmen, gibt es einen Teil an Minijobberinnen, die insofern nicht freiwillig einen Minijob angenommen haben, weil sie aufgrund von beispielsweise geringer Bildung keine sozialversicherungspflichtige Tätigkeit finden konnten, Minijobs also wegen fehlender Alternativen ausüben (Klenner/Schmidt 2012). Befragungen zeigen, dass gerade geringfügig tätige Frauen nicht selten an einer Ausweitung ihrer Arbeitszeit interessiert sind (vgl. Wanger/Weber 2014).

Übergänge von geringfügiger in sozialversicherungspflichtige Beschäftigung werden durch verschiedene Faktoren erschwert. Ausschließlich geringfügige Beschäftigung ist im Gegensatz zur sozialversicherungspflichtigen Beschäftigung nicht steuerpflichtig. Bei Sozialleistungsbeziehenden werden höhere Einkommen aus Erwerbsarbeit in der Arbeitslosenversicherung und Grundsicherung in starkem Maße angerechnet. Zudem ist das Angebot flexibler und gleichzeitig bezahlbarer Betreuungskapazitäten für Kinder und Pflegebedürftige noch immer eingeschränkt. Verlaufsuntersuchungen spiegeln diese gravierende Übergangsproblematik wider. Zwar gibt es den Befund, dass Minijobs die Verbleibschancen am Arbeitsmarkt, einschließlich des Verbleibs in geringfügiger Beschäftigung, erhöhen (Caliendo/Wrohlich 2010). Jedoch finden sich nur wenig Hinweise für Minijobs als Brücke in reguläre Beschäftigung (Dingeldey et al. 2012). Auch unter Berücksichtigung persönlicher Lebensentwürfe und Ar-

4 Dabei zeigen sich jedoch nach Geschlecht beträchtliche Unterschiede. Während der Anteil der teilzeitbeschäftigten Männer, die keine Vollzeittätigkeit finden konnten, 2013 be $25 \%$ lag, betrug der entsprechende Anteil bei den Frauen $14 \%$. Dies fällt aber deshalb weniger ins Gewicht, weil Männer in geringerem Maße in derTeilzeitbeschäftigung vertreten sind. 
beitszeitpräferenzen zeigen sich deutlich geringere Übergangschancen von geringfügig Beschäftigten in ein Normalarbeitsverhältnis im Vergleich zu anderen atypischen Erwerbsformen (Brülle 2013). Des Weiteren zeichnen sich Minijobs durch eine vergleichsweise hohe Fluktuation aus. Betrachtet man die Gruppe der ausschließlich geringfügig entlohnten Beschäftigten, ist die Fluktuation doppelt so hoch wie bei sozialversicherungspflichtig Beschäftigten (Kalina/Voss-Dahm 2005).

Die Entwicklung geringfügiger Nebenjobs unterscheidet sich von der ausschließlich geringfügig Tätiger. Geringfügige Nebenjobs verzeichnen zwischen 2005 und 2013 ein deutliches Plus von 844.000 oder 56,7\%. Ihr Zuwachs beeinflusst jedoch nicht direkt die Zahl der Erwerbstätigen, sondern impliziert eine individuelle Arbeitszeitverlängerung. Oft kommt darin der Wunsch eines Zusatzverdienstes zum Ausdruck (Schmidt/Voss 2014), der durch die Beschäftigungsform auf flexible Weise ermöglicht wird. Dagegen wäre eine bezahlte Mehrarbeit, so sie denn überhaupt realisiert werden könnte, im Hauptbeschäftigungsverhältnis mit höheren Abgaben verbunden. Ordnungspolitisch ist die Regelung problematisch, weil sie fragwürdige Anreize in Richtung einer steuerlich begünstigten Zweitbeschäftigung setzen kann.

\subsection{Befristete Arbeitsverträge: bei Einstellungen fast schon die Regel}

Der Anteil befristeter Arbeitsverträge an der abhängigen Beschäftigung hat seit 2005 leicht abgenommen. Dies gilt in gleichem Maße für die Befristungen insgesamt (- 0,8 Prozentpunkte) wie für vollzeitnahe Befristungen mit einer wöchentlichen Arbeitszeit von mehr als 31 Stunden (-0,8 Prozentpunkte). Gegenüber der Zeit vor 2005 hat sich das Wachstum der Befristungen spürbar verringert. Der Anstieg ist bei den Befristungen insgesamt von 27,8\% im Zeitraum 1997/2005 auf $1 \%$ in der Periode 2005/2013 deutlich gefallen. Bei den vollzeitnahen Befristungen zeigt sich bei den entsprechenden Werten ebenfalls eine massive Veränderung: Dem Wachstum vor 2005 von 16,7\% folgte ein Rückgang in der Zeit nach 2005 von 3,5\%. Die Differenzen zwischen beiden Befristungskategorien legen nahe, dass befristete Arbeitsverträge nicht selten mit Teilzeitbeschäftigung einhergehen. Die große Bedeutung von Befristungen am Arbeitsmarkt kommt zudem in dem relativ hohen Anteil befristeter Einstellungen an allen Einstellungen zum Ausdruck, der sich seit 2005 in einem Bereich von um die $45 \%$ bewegt - mit zuletzt leicht rückläufiger Tendenz (Hohendanner 2013). Die höchsten Befristungsquoten bei den Einstellungen verzeichneten dabei die Wirtschaftsbereiche Erziehung und Unterricht (76\%), Organisationen ohne Erwerbscharakter (68\%) und öffentliche Verwaltung (60\%). Befristungen wurden in den letzten Jahren häufig als eine Art verlängerte Probezeit genutzt, was sich auch in teils beträchtlichen und vom Arbeitsmarkttrend abhängigen Übernahmequoten zeigt. Sie sind zwischen 2009 und 2013 von 30 auf $37 \%$ gestiegen.
Für die Einschätzung der Rolle befristeter Beschäftigung am Arbeitsmarkt ist wiederum die Frage nach den möglichen Alternativen für die Betroffenen zu stellen. Dabei liefert der Mikrozensus keine Hinweise auf einen wachsenden Anteil von Personen, die keine Dauerstellung finden konnten. Der entsprechende Anteil an allen Befristungen ist seit 2000 von 22,1 zwar auf 25,6\% im Jahr 2005 gestiegen, mit der Verbesserung am Arbeitsmarkt nach 2005 jedoch auf 19,4\% im Jahr 2013 gesunken. Andererseits sind auch nur gut $2 \%$ der befristet Beschäftigten nicht an einer Dauerstelle interessiert. Die Bedeutung von Befristungen mit der Funktion eines Probearbeitszeitvertrags ist aus Beschäftigtensicht nahezu gleich geblieben (2000: $21 \%, 2013: 22 \%$ ).

Bezüglich der Sprungbrettfunktion befristeter Beschäftigung zeigt Gebel (2013), dass die Aufnahme eines befristeten Jobs in den fünf Folgejahren im Vergleich zum Verbleib in der Arbeitslosigkeit die Beschäftigungschancen allgemein erhöht und den Zugang zu einer unbefristeten Stelle erleichtert. Auch im Vergleich zu anderen atypischen Erwerbsformen weisen befristet Beschäftigte die besten Chancen auf, in ein Normalarbeitsverhältnis zu münden (Brülle 2013). Naheliegenderweise zeigen befristet Beschäftigte ein hohes Maß an Veränderungsbereitschaft bzw. -bedarf, denn laut Mikrozensus sind sie häufiger auf der Suche nach einer anderen Tätigkeit als beispielsweise unbefristet Vollzeit- oder Teilzeitbeschäftigte (Himsel/Walwei 2015). Allerdings muss dabei zwischen den überproportional betroffenen Akademikern und Geringqualifizierten unterschieden werden: Während bei Akademikern häufig nur der Berufseinstieg durch befristete Arbeitsverhältnisse gekennzeichnet ist, sind bei Geringqualifizierten wesentlich häufiger Drehtüreffekte zu beobachten (Achatz et al. 2012).

\subsection{Leiharbeit: Stagnation nach Wachstum}

Neben den Minijobs ist Leiharbeit die Beschäftigungsform mit dem stärksten Anstieg in den letzten anderthalb Dekaden. Dies zeigen die besonders ausgeprägten Wachstumsraten in den beiden Perioden 1997/2005 (+112,7\%) und 2005/2013 (+88,1\%). Besonders in den Jahren unmittelbar nach der Hartz-Reform (2003), die eine umfassende Deregulierung der Arbeitnehmerüberlassung mit sich brachte, ergab sich von 2003 bis 2007 ein starker Zuwachs von 327.000 auf 731.000, sodass sich die Zahl der Leiharbeitnehmer mehr als verdoppelte. Eine Niveauverschiebung der Zeitarbeit nach oben ist nach der großen Reform damit klar zu konstatieren. Allerdings nahm die Wachstumsdynamik in den Folgejahren ab, denn 2013 lag die Zahl der Leiharbeitnehmer mit 852.000 nur unwesentlich höher als 2007. Viele Gründe kommen für die zuletzt weniger aufwärtsgerichtete Entwicklung der Zeitarbeitsbranche in Betracht: Neben einzelnen Regulierungsinstrumenten, wie dem Branchenmindestlohn sowie Beschränkungen bei der Konzernleihe, haben sich für Arbeitnehmer durch den Aufschwung am Arbeitsmarkt die Chancen außerhalb der 
Zeitarbeitsbranche verbessert. Auch ein Ausweichen auf die zuletzt häufiger zu beobachtenden Werkverträge zwischen Unternehmen könnte den Anstieg der Leiharbeit gebremst haben.

Für eine beschäftigungspolitische Einschätzung der Erwerbsform sind zwei Aspekte von besonderer Bedeutung. Zum einen sind Arbeitsverhältnisse in der Zeitarbeitsbranche im Durchschnitt eher kurz. Ein Großteil dauert nur wenige Monate und ist durch eine hohe Fluktuation gekennzeichnet (Haller/Jahn 2014; Möller et al. 2012). Für die Betroffenen geht dies mit einer beträchtlichen Beschäftigungsunsicherheit einher. Zweitens sind substanzielle Brückeneffekte der Leiharbeit in eine Beschäftigung außerhalb der Zeitarbeitsbranche nicht erkennbar (Lehmer 2012). Eine wichtige, aber noch nicht hinlänglich beantwortete Frage ist dabei, ob dafür die in der Tendenz schwächere Humankapitalausstattung der in der Zeitarbeitsbranche tätigen Personen im Vergleich zu Normalbeschäftigten oder Einsperr- und Drehtüreffekte der Beschäftigungsform maßgeblich sind (Dütsch 2011).

\subsection{Geringverdiener: langfristiger Aufwärtstrend setzt sich abgeschwächt fort}

Der Anteil der Beschäftigten mit niedrigen Stundenlöhnen ist im Zuge des Aufschwungs am Arbeitsmarkt offenbar nur noch leicht gewachsen. Auf der Basis des Sozio-oekonomischen Panels weisen Kalina und Weinkopf (2014) ausgehend von einem Schwellenwert von Zweidrittel des Medianstundenlohns einen Niedriglohnanteil für 2012 von 24,3\% für Gesamtdeutschland aus, der etwas höher liegt als der entsprechende Prozentwert im Jahr 2005 von 23,1 \%. Der größte Teil des Zuwachses im Niedriglohnsektor vollzog sich demnach in der Zeit vor 2005. Allein zwischen 1997 und 2005 legte die Quote der Geringverdiener um mehr als drei Prozentpunkte zu. Die Befunde zeigen weiter eine starke Korrelation mit atypischer Beschäftigung. Denn niedrige Stundenlöhne betreffen häufiger sozialversicherungspflichtige Teilzeitbeschäftigung und noch mehr Minijobs. Darüber hinaus ergeben Analysen zu Befristungen und Leiharbeit teils beträchtliche Bruttolohndifferenzen im Vergleich zur Normalbeschäftigung (Keller/Seifert 2013)

Die hohe Inzidenz von Niedriglohnbeschäftigung ist als ein klarer Hinweis auf Lohnungleichheit in der Beschäftigung zu sehen. Ein anhaltend hoher Niedriglohnsektor führt zudem dazu, dass sich bei Menschen, die länger im gering entlohnten Segment verbleiben, die Höhe der individuellen Rentenanwartschaften nachhaltig verringert (Leiber 2009). Viele Niedriglohnberufe befinden sich im Dienstleistungssektor und werden häufig von Frauen ausgeübt. Auch der hohe Anteil teilzeitbeschäftigter Frauen im Niedriglohnbereich macht Geschlechterungleichheiten deutlich (George 2011). Das Risiko, im Niedriglohnbereich beschäftigt zu sein, variiert insgesamt zwischen verschiedenen Personengruppen: gering Qualifizierte, befristet Beschäftigte und Ausländer sind unter den Geringverdienern überrepräsentiert (Rhein 2013).
Für eine weitergehende Beurteilung sind zwei zusätzliche Aspekte von Bedeutung. Niedriglohnjobs sind aus Verteilungssicht noch problematischer, wenn sie für die Betroffenen und ihre jeweiligen Haushalte die einzige Einkommensquelle darstellen. Sind sie dagegen eher ein Zusatzeinkommen, relativiert sich das Risiko für den betreffenden Haushalt, weil noch andere Einnahmequellen für den Lebensunterhalt zur Verfügung stehen können (Göbel et al. 2005). Der andere Aspekt betrifft die Aufstiegschancen in höhere Lohnsegmente. Je mehr Niedriglohnbeschäftigung als Brücke in höher bezahlte Tätigkeiten fungiert, desto eher wächst deren Akzeptanz als Einstiegsoption in den Arbeitsmarkt (Stephani 2012; Mosthaf 2011). So zeigen Studien, die sich auf westdeutsche Männer in Vollzeit konzentrieren, gerade bei den am schlechtesten bezahlten Geringverdienern ein gewisses Ausmaß an Aufwärtsmobilität im Niedriglohnsektor, die dann auch über mehrere Jahre anhält. Andere Studien machen wiederum die Persistenz und geringe Aufstiegschancen aus dem Niedriglohnsektor deutlich: Gerade bei Frauen und bei Älteren waren in der zweiten Hälfte der letzten Dekade die Chancen der Aufwärtsmobilität vom Niedriglohnbereich in eine bessere Bezahlung vergleichsweise gering (Aretz/ Gürtzgen 2012; Mosthaf et al. 2009; Uhlendorff 2006).

\subsection{Aufstocker: Chancen und Risiken}

Ergänzende Sozialleistungen für Erwerbstätige gab es bereits in dem vor 2005 getrennten System von Arbeitslosen- und Sozialhilfe und zwar in ähnlicher Höhe wie nach der Einführung des Sozialgesetzbuches (SGB) II im Jahre 2005 (Rudolph 2014). In den ersten Jahren nach der Reform legte die Zahl der erwerbstätigen Hilfebedürftigen (Aufstocker) bis etwa 2008 zunächst kräftig zu. Von anfänglich 760.000 Aufstockern stieg deren Zahl zunächst auf etwa 1,3 Mio. an und stagniert seitdem. Allerdings wuchs nach 2008 der Aufstockeranteil an allen Hilfebedürftigen, weil die Zahl der Grundsicherungsempfänger abnahm. Der Bedarf an ergänzenden Leistungen für erwerbstätige Hilfebedürftige hängt dabei insbesondere von deren jeweiligen Stundenlöhnen, Arbeitszeiten und dem jeweils relevanten Haushaltskontext ab (Bruckmeier et al. 2013).

Der Großteil der abhängig beschäftigten Aufstocker arbeitet in Teilzeit, wodurch deren Verdienstpotenzial eingeschränkt wird. So arbeiten fast $90 \%$ der Aufstocker weniger als 31 Stunden in der Woche. Die Stundenlöhne hilfebedürftiger Erwerbstätiger waren in 2011 mit durchschnittlich 6,20€ brutto in der Stunde eher niedrig. Zudem sind Alleinstehende und Alleinerziehende gemessen an ihrem Bevölkerungsanteil unter den Aufstockern überrepräsentiert. Im Vergleich zu Paarhaushalten erreichen beide Gruppen insbesondere wegen der Kombination aus einer relativ niedrigen Zahl von Arbeitsstunden und tendenziell geringeren Stundenlöhnen häufig kein existenzsicherndes Einkommen. Bei den vollzeitbeschäftigten Aufstockern begrenzt vor allem die größere Zahl von Personen (knapp drei Viertel), die in Paarhaushalten mit und 
ohne Kinder leben, das Erreichen des soziokulturellen Existenzminimums stärker.

Ausstiegsmöglichkeiten und Verbleib im Aufstockerstatus werden durch spezifische Faktoren beeinflusst (Bruckmeier et al. 2013). Ein höherer Berufsabschluss und ein bereits erzielter hoher Stundenlohn, weniger Kinder und mehr erwerbsfähige Personen begünstigen den Weg aus dem Leistungsbezug. Eher hinderlich sind dagegen mangelnde berufliche Qualifikation und schwere gesundheitliche Einschränkungen (Dietz et al. 2009). Auch unzureichende Kinderbetreuungsmöglichkeiten können einer Ausweitung der Arbeitszeit entgegenstehen. In solchen Fällen kann aus individueller Perspektive selbst der Aufstockerstatus im Vergleich zur Nichterwerbstätigkeit als Erfolg betrachtet werden und nicht nur als Problem einer mangelnden Existenzsicherung. Immerhin schaffen diese Leistungsbezieher eine - wenn auch nur partielle - Integration in den Arbeitsmarkt und damit einen Beitrag zu ihrem Lebensunterhalt. Die ausgeübte Erwerbstätigkeit kann mittelfristig die individuellen Chancen erhöhen, den Leistungsbezug zu beenden.

\section{Fazit}

Unsere Ergebnisse zeigen, dass die Entwicklung des Arbeitsmarktes nach der Trendwende einer außerordentlich differenzierten Betrachtung bedarf. Zunächst einmal ist bemerkenswert, in welch starkem Maße die Erwerbstätigkeit insgesamt gestiegen ist und damit ihren Anteil an der Bevölkerung im erwerbsfähigen Alter deutlich steigern konnte. Von besonderer Bedeutung ist auch, dass der Aufwuchs der sozialversicherungspflichtigen Beschäftigung wesentlicher Treiber des Jobbooms war. Dabei darf zwar nicht außer Acht gelassen werden, dass sich die sozialversicherungspflichtige Beschäftigung aus heterogenen Erwerbsformen zusammensetzt. Denn darunter fallen u.a. Teilzeitbeschäftigungsverhältnisse, befristete Arbeitsverträge, Leiharbeitnehmer und Vollzeitarbeitsverhältnisse mit niedrigem Stundenlohn. Festzuhalten ist aber, dass am aktuellen Rand wesentlich mehr Menschen über Erwerbsteilhabe Ansprüche auf soziale Leistungen erwerben als dies in den letzten beiden Dekaden der Fall war.

Auch die Beantwortung der Frage nach den mit der Trendwende möglicherweise verbundenen Schattenseiten bedarf eines genauen Blicks auf die Fakten. Das Normalarbeitsverhältnis hat sich im Zuge des Umschwungs nicht nur in absoluten Größen erholt, sondern legte zuletzt anteilsmäßig - wenn auch nur leicht - wieder zu. Dies legt nahe, dass sich der Trade-off zwischen Quantität und Qualität der Beschäftigung in der jüngeren Vergangenheit etwas gelockert hat. Verstärkt wird diese Beobachtung dadurch, dass die wachsende Ungleichheit in der Beschäftigung kein neues Phänomen ist, das erst im Zuge der jüngeren Arbeitsmarktentwicklungen und -politiken entstanden wäre. Die Strukturveränderungen am Arbeitsmarkt repräsentieren vielmehr auch eine längerfristige Entwicklung zugunsten flexibler Formen der Beschäftigung. Aber mehr noch, seit 2005 haben die Veränderungen im Ganzen betrachtet an Dynamik verloren. Die Wachstumsraten von atypischer Beschäftigung und Niedriglohnbeschäftigung fallen zwischen 2005 und 2013 tendenziell geringer aus als in der Vorperiode 1997 bis 2005. In Teilen zeigt sich ähnlich wie bei den Normalarbeitsverhältnissen zuletzt eine gewisse Stagnation der Entwicklung. Eine mögliche Ursache dafür könnte die allgemein verbesserte Arbeitsmarktlage sein, welche die Marktmacht der Erwerbspersonen gestärkt und damit den $\mathrm{Zu}$ - gang in eine stabile und existenzsichernde Beschäftigung insgesamt erleichtert hat. Eine Rolle könnten dabei auch die von der aktuellen und der vorherigen Bundesregierung eingeleiteten Re-Regulierungen spielen, die auf eine Stärkung der Arbeitnehmerrechte zielten (z. B. die Einführung sektoraler Mindestlöhne).

Auch wenn mögliche Schattenseiten der Trendwende am Arbeitsmarkt angesichts der empirischen Befunde zu relativieren sind, gibt es noch Handlungsbedarf. Zwar gab es nach der Trendwende am Arbeitsmarkt keine wesentliche Verschlechterung der Beschäftigungsstruktur mehr, aber auch keine Verbesserung. Zumindest bis dato konnte der kräftige Jobboom den Anteil atypischer Beschäftigungsverhältnisse und niedrig entlohnter Tätigkeiten nicht spürbar reduzieren. Ein wesentliches Problem besteht hier darin, dass flexible und niedrig entlohnte Formen der Beschäftigung gerade für Geringqualifizierte und andere Problemgruppen des Arbeitsmarktes zwar den Einstieg in die Beschäftigung erleichtern, aber selten eine Brücke in eine stabile und besser bezahlte Tätigkeit schlagen. Ein Ansatzpunkt, um der Ungleichheit in der Beschäftigung entgegenzuwirken, sind berufsbegleitende und abschlussorientierte Weiterbildungsaktivitäten, die sich positiv auf Erwerbskarrieren auswirken können (Dietz et al. 2013).

Schließlich darf der Wandel der Erwerbsformen nicht pauschal mit einem Zuwachs an „bad jobs“ gleichgesetzt werden. Im Einzelfall sind Fragen der Freiwilligkeit, der möglichen Alternativen, der erwerbsbiografischen Situation und des Haushaltskontexts zu berücksichtigen. Eine zusätzliche Frage in diesem Kontext ist insofern, ob - gemessen an früheren Zeiten und gerade unter Einschluss der Alternative Arbeitslosigkeit - prekäre Erwerbsbiografien zugenommen haben. An dieser Stelle gibt es weiteren Forschungsbedarf, der den längerfristigen Erwerbsverlauf in den Mittelpunkt stellen muss.

\section{LITERATUR}

Achatz, J./Bruckmeier, K./Buch, T./Burkert, C./Dietrich, H./Dietz, M. et al. (2012): Übergänge am Arbeitsmarkt und Qualität von Beschäftigung, in: Brücker, H./Klinger, S./Möller, J./Walwei, U. (Hrsg.): Handbuch Arbeitsmarkt 2013. Analysen, Daten, Fakten, IAB-Bibliothek, Bd. 334, Bielefeld, S. 141-203 Andreß, H.-J./Seek, T. (2007): Ist das Normalarbeitsverhältnis armutsvermeidend?, in: Kölner Zeitschrift für Soziologie und Sozialpsychologie 59 (3), S. 459-492

Aretz, B./Gürtzgen N. (2012): What explains the decline in wage mobility in the German low-wage sector?, ZEW Discussion Paper 12-041, Mannheim Belke, A./Göcke, M. (1994): Starke Hysteresis auf dem Arbeitsmarkt, in: ZWS-Zeitschrift für Wirtschafts- und Sozialwissenschaften 114 (3), S. 345-377 Bosch, G. (2012): Prekäre Beschäftigung und Neuordnung am Arbeitsmarkt, Expertise im Auftrag der IG Metall, Duisburg

Brehmer, W./Seifert, H. (2008): Sind atypische Beschäftigungsverhältnisse prekär? Eine empirische Analyse sozialer Risiken, in: Zeitschrift für ArbeitsmarktForschung 41 (4), S. 501-531

Bruckmeier, K./Eggs, J./Himsel, C./Trappmann, M./Walwei, U. (2013):

Aufstocker im SGB II: Steinig und lang - der Weg aus dem Leistungsbezug, IAB-Kurzbericht (14), Nürnberg

Brülle, J. (2013): Unterschiede in den Arbeitsmarktchancen von atypisch Beschäftigten: Effekte von Beschäftigungsformen oder Erwerbspräferenzen?, in: Zeitschrift für Soziologie 42 (2), S. 157-179

Caliendo, M./Wrohlich, K. (2010): Evaluating the German 'Mini-Job' reform using a natural experiment, in: Applied Economics 42 (19), S. 2475-2489 Caliendo, M./Hogenacker, J./Künn, S./Wießner F. (2012): Alte Idee, neues Programm: Der Gründungszuschuss als Nachfolger von Überbrückungsgeld und Ich-AG, in: Journal for Labour Market Research 45 (2), S. 99-123 DGB (Deutscher Gewerkschaftsbund) (2013): Atypische und prekäre Beschäftigung weiterhin auf hohem Niveau, Arbeitsmarkt auf den Punkt gebracht (5), Berlin

Dietz, M./Himsel, C./Walwei, U. (2013): Wandel der Erwerbsformen: welche Rolle spielen strukturelle Änderungen am Arbeitsmarkt?, in: Arbeit. Zeitschrift für Arbeitsforschung, Arbeitsgestaltung und Arbeitspolitik 22 (2), S. 85-104 
Dietz, M./Müller, G./Trappmann, M. (2009): Bedarfsgemeinschaften im SGB II: Warum Aufstocker trotz Arbeit bedürftig bleiben, IAB-Kurzbericht 2/2009, Nürnberg

Dietz, M./Stops, M./Walwei, U. (2012): Vollbeschäftigung in Sicht? Zur Lage auf dem Arbeitsmarkt, in: Aus Politik und Zeitgeschichte 62 (14/15), S. 20-30 Dingeldey, I./Sopp, P./Wagner, A. (2012): Governance des Einkommensmix: Geringfügige Beschäftigung im ALG-II-Bezug, in: WSI-Mitteilungen 65 (1), S. 32-40, http://www.boeckler.de/wsi-mitteilungen_38633_38650.htm Dustmann, C./Fitzenberger, B./Schönberg, U./Spitz-Oener, A. (2014): From sick man of Europe to economic superstar: Germany's resurgent economy, in:The Journal of Economic Perspectives 28 (1), S. 167-188 Dütsch, M. (2011): Wie prekär ist Zeitarbeit? Eine Analyse mit dem MatchingAnsatz, in: Zeitschrift für ArbeitsmarktForschung 43 (4), S. 299-318 Fuchs, J./Hummel, M./Hutter, C./Klinger, S./Wanger, S./Weber, E./Weigand, R./Zika, G. (2014): Arbeitsmarkt 2014/2015: Robust, aber risikobehaftet, IAB-Kurzbericht 18/2014, Nürnberg

Gebel, M. (2013): Is a temporary job better than unemployment? A crosscountry comparison based on British, German, and Swiss panel data, SOEPpapers (543), Berlin

George, R. (2011): Niedriglohn und Geschlecht im europäischen Vergleich, in: WSI-Mitteilungen 64 (10), S. 548-555, http://www.boeckler.de/wsi-mitteilungen 38011_38018.htm

Göbel, J./Krause, P./Schupp, J. (2005): Mehr Armut durch steigende Arbeitslosigkeit: Niedriglöhne überwiegend als Zusatzeinkommen im Haushalt, in: DIW Wochenbericht 72 (10), S. 175-184

Haller, P./Jahn, E. (2014): Zeitarbeit in Deutschland: Hohe Dynamik und kurze Beschäftigungsdauern, IAB-Kurzbericht 13/2014, Nürnberg

Himsel, C./Walwei, U. (2015): Determinanten der Arbeitsuche atypisch Beschäftigter, in: Arbeit. Zeitschrift für Arbeitsforschung, Arbeitsgestaltung und Arbeitspolitik 23 (3), S. 225-241

Hohendanner, C. (2013): Befristete Beschäftigung - Aktuelle Zahlen aus dem IAB-Betriebspanel 2012, Nürnberg

Hohendanner, C./Gundert, S. (2011): Leiharbeit und befristete Beschäftigung: Soziale Teilhabe ist eine Frage von stabilen Jobs, IAB-Kurzbericht 4/2011, Nürnberg

Holst, E./Seifert, H. (2012): Arbeitszeitpolitische Kontroversen im Spiegel der Arbeitszeitwünsche, in: WSI-Mitteilungen 65 (2), S. 141-149, http://www.boeckler.de/wsi-mitteilungen_39052_39060.htm

Kalina, T./Voss-Dahm, D. (2005): Mehr Minijobs = mehr Bewegung auf dem Arbeitsmarkt? Fluktuation der Arbeitskräfte und Beschäftigungsstruktur in vier Dienstleistungsbranchen, IAT-Report 2005-07, Gelsenkirchen Kalina,T./Weinkopf, C. (2014): Niedriglohnbeschäftigung 2012 und was ein gesetzlicher Mindestlohn von 8,50€ verändern könnte, in: IAQ-Report 2014-02, Duisburg

Keller, B./Seifert, H. (2013): Atypische Beschäftigung zwischen Prekarität un Normalität: Entwicklung, Strukturen und Bestimmungsgründe im Überblick, Berlin

Klenner, C. (2011): Prekarisierung der Arbeit - Prekarisierung im Lebenszusammenhang, Editorial, in:WSI-Mitteilungen 64 (8), S. 378,

http://www.boeckler.de/wsimit_2011_08 editorial.pdf

Klenner, C./Schmidt, T. (2012): Minijobs - Eine riskante Beschäftigungsform beim normativen Übergang zum "Adult-Worker-Model“, in: WSI-Mitteilungen 65 (1), S. 22-31, http://www.boeckler.de/cps/rde/xchg/hbs/hs.xsl/wsimitteilungen_38633_38651.htm

Koller, L./Neder, N./Rudolph, H./Trappmann, M. (2012): Selbstständige in der Grundsicherung: Viel Arbeit für wenig Geld, IAB-Kurzbericht 22/2012, Nürnberg

Körner, T./Meinken, H./Puch, K. (2013): Wer sind die ausschließlich geringfügig Beschäftigten? Eine Analyse nach sozialer Lebenslage, in: Wirtschaft und Statistik (01) Januar, S. 42-61

Krugman, P. (1994): Past and prospective causes of high unemployment, in: Economic Review - Federal Reserve Bank of Kansas City 79 (4), S. 23-43

Lehmer, F. (2012): Dient die Arbeitnehmerüberlassung für Langzeitarbeitslose als Brücke in nachhaltige Beschäftigung?, in: Sozialer Fortschritt 61 (8), S. $190-197$

Leiber, S. (2009): Armutsvermeidung im Alter: Handlungsbedarf und Handlungsoptionen, WSI-Diskussionspapiere (166), Düsseldorf

Möller, J./Völker, R. (1991): Lohnbildung und Hysteresis. Empirische Überprüfung eines Insider-Outsider-Modells für die Bundesrepublik Deutschland, in: Zeitschrift für Wirtschafts- und Sozialwissenschaften 111 (3), S. $401-424$
Möller, J./Walwei, U./Ziegler, K. (2012): Wie wichtig ist Zeitarbeit für den deutschen Arbeitsmarkt?, in: Dinges, A./Franken, H./Breucker, G./Calasan, V./Speidel, C. (Hrsg.): Zukunft Zeitarbeit. Perspektiven für Wirtschaft und Gesellschaft, Berlin, S. 33-53

Mosthaf, A. (2011): Low-wage jobs - stepping stones or just bad signals?, IAB Discussion Paper 11/2011, Nürnberg

Mosthaf, A./Schank, T./Schnabel, C. (2009): Low-wage employment versus unemployment: Which one provides better prospects for women?, IZA Discussion Paper (4611), Bonn

OECD (Organisation für wirtschaftliche Zusammenarbeit und Entwicklung) (2014): OECD-Wirtschaftsberichte: Deutschland 2014, OECD Publishing Posen, A. S. (2013): Deutschlands Exportsucht zerstört die Euro-Zone, in: Die Welt vom 14.06., http://www.welt.de/wirtschaft/article117134282/ Deutschlands-Exportsucht-zerstoert-die-Euro-Zone.html, (letzter Zugriff: 02.02.2015)

Rhein, T. (2013): Erwerbseinkommen: Deutsche Geringverdiener im europäischen Vergleich, IAB-Kurzbericht 15/2013, Nürnberg

Rodgers, G. (1989): Precarious work in Western Europe, in: Rodgers G./ Rodgers, J. (Hrsg.): Precarious jobs in labour market regulation. The growth of atypical employment in Western Europe, Genf, S. 1-16

Rudolph, H. (2014): „Aufstocker": Folge der Arbeitsmarktreformen?, in: WSI-Mitteilungen 67 (3), S. 207-217, http://www.boeckler.de/wsi-mitteilungen_ 46852 46861.htm

Schmidt, T./Voss, D. (2014): Arbeitsmarkt- und geschlechtsdifferenzielle Einflussfaktoren für die Ausübung einer geringfügigen Nebenbeschäftigung, in: Industrielle Beziehungen 21 (1), S. 36-57

Statistik der Bundesagentur für Arbeit (2014a): Arbeitsmarkt in Zahlen Arbeitsmarktstatistik: Arbeitslosenquoten 2013, Nürnberg

Statistik der Bundesagentur für Arbeit (2014b): Der Arbeitsmarkt in Deutschland, Frauen und Männer am Arbeitsmarkt im Jahr 2013, Nürnberg Statistisches Bundesamt (2012): Qualität der Arbeit. Geld verdienen und was sonst noch zählt 2012, Wiesbaden

Stephani, J. (2012): Wage growth and career patterns of German low-wage workers, IAB-Discussion Paper 1/2012, Nürnberg

Uhlendorff, A. (2006): From no pay to low pay and back again? A multi-state model of low pay dynamics, IZA Discussion Paper (2482), Bonn

Walwei, U. (2011): Veränderte Strukturen des deutschen Arbeitsmarktes: zukunftsfähig oder doch nicht nachhaltig?, in: WSI-Mitteilungen 64 (11), S. 563-570, http://www.boeckler.de/wsi-mitteilungen_38168_38179.htm Wanger, S. (2011): Ungenutzte Potenziale in derTeilzeit: Viele Frauen würden gerne länger arbeiten, IAB-Kurzbericht 9/2011, Nürnberg

Wanger, S./Weber, E. (2014): Arbeitszeitwünsche von Frauen und Männern 2012, IAB - Aktuelle Daten und Indikatoren, Nürnberg

Weber, E. (2014): Das Ziel der Vollbeschäftigung in Deutschland: Fern, aber erreichbar, IAB-Kurzbericht 15/2014, Nürnberg

Wingerter, C. (2009): Der Wandel der Erwerbsformen und seine Bedeutung für die Einkommenssituation Erwerbstätiger, in: Wirtschaft und Statistik $11 / 2009$, S. $1080-1098$

\section{AUTOREN}

CARINA SPERBER, Sozialökonomin (M.Sc.), ist Wissenschaftliche Mitarbeiterin beim Vizedirektor des Instituts für Arbeitsmarkt- und Berufsforschung (IAB), Nürnberg. Arbeitsschwerpunkte: Atypische Beschäftigung, Wandel der Erwerbsformen, erwerbstätige Leistungsbezieher im SGB II ("Aufstocker“).

carina.sperber@iab.de

ULRICH WALWEI, Dr. rer. pol., ist Vizedirektor des IAB. Arbeitsschwerpunkte: Institutionenökonomie, insbesondere Fragen der Flexibilisierung und Deregulierung des Arbeitsmarkts, längerfristigen Trends der Beschäftigung und der Zukunft des Arbeitsmarktes.

ulrich.walwei@iab.de 